\title{
BMJ Open Barriers in health and social care access and navigation for elder orphans: a scoping review protocol
}

\author{
Lucy Kervin (D) , ${ }^{1}$ Kelly Teo (D) , ${ }^{1}$ Ryan Churchill (D) , ${ }^{1}$ Indira Riadi (D) , \\ Theodore D Cosco (1) 1,2
}

To cite: Kervin L, Teo K, Churchill $\mathrm{R}$, et al. Barriers in health and social care access and navigation for elder orphans: a scoping review protocol. BMJ Open 2021;11:e043876. doi:10.1136/ bmjopen-2020-043876

- Prepublication history for this paper is available online. To view these files, please visit the journal online (http://dx.doi. org/10.1136/bmjopen-2020043876).

Received 16 August 2020 Accepted 05 May 2021

Check for updates

(C) Author(s) (or their employer(s)) 2021. Re-use permitted under CC BY-NC. No commercial re-use. See rights and permissions. Published by BMJ.

${ }^{1}$ Gerontology Research Centre, Department of Gerontology,

Simon Fraser University,

Vancouver, British Columbia,

Canada

${ }^{2}$ Institute of Population Ageing, University of Oxford, Oxford,

Oxfordshire, UK

Correspondence to

Lucy Kervin; lucy_kervin@sfu.ca

\section{ABSTRACT}

Introduction Family members have traditionally been relied on to provide informal care to older adults. However, social and demographic changes are resulting in rising numbers of kinless and isolated elderly who are unable to rely on familial caregiving and are without assistance in navigating complex systems of health and social services. Research examining this vulnerable subset of the elderly population, identified as elder orphans, is limited, particularly within the context of health and social care access. The aim of this scoping review is to map and report the evidence available in identifying barriers and facilitating factors in health and social care access and system navigation by elder orphans.

Methods and analysis Arksey and 0'Malley's sixstaged methodology framework will guide the conduct of this scoping review. The primary author will conduct a systematic search and an initial screen of titles and abstracts from six electronic databases (CINAHL Complete, ASSIA, Pubmed, Scopus, Web of Science and PsycINF0) from January 2005 to the date of commencement, to identify English language peer reviewed studies of various methodologies. Subsequently, two reviewers will independently screen a shorter list of studies for inclusion. We will also search the reference lists of eligible studies. Data from the selected studies will be extracted and charted by two independent reviewers. Findings will be summarised in a tabulated format and accompanied by a narrative synthesis.

Ethics and dissemination As the scoping review methodology aims to synthesise information from publicly available materials, this study does not require ethical approval. The finalised scoping review will be submitted for publication to a scientific journal.

Registration The present protocol has been registered with the Open Science Framework platform (registration ID: https://osf.io/7vjty/).

\section{INTRODUCTION}

The support of close kin is deeply implicated in processes of healthy ageing, through provision of both socioemotional and tangible resources. Familial ties serve to combat social isolation ${ }^{1}$ and loneliness, ${ }^{2}$ reinforce senses of meaning and purpose, ${ }^{3}$ and further supply the foundation for informal caregiving for the elderly. ${ }^{4}$ Family members adopt

\section{Strengths and limitations of this study}

- The proposed scoping review will map and synthesise current knowledge on barriers in health and social care access and system navigation for elder orphans; to the best of our knowledge, this is the first review of its kind on this important topic.

- We will conduct a comprehensive literature search of multiple electronic databases using iteratively refined, tailored search strings to increase the likelihood of retrieving as many relevant articles as possible.

- Our scoping review will conform to the methodologically rigorous methods put forth by the Joanna Briggs Institute and will be guided by Arksey and 0'Malley's eminent work on scoping reviews.

- In consideration of the inception of this emergent topic taking place in the early 2000s and to reflect current health and social care systems and practices, we will limit our search to English peer-reviewed papers disseminated in the past 15 years.

- As quality assessment of the articles chosen for inclusion will not be performed, the findings from this review will be subject to the strengths and limitations of the included studies.

innumerable responsibilities as the health of their elderly relations declines, ranging from assistance with personal care and daily activities, administrative tasks and monitoring of clinical symptoms and medications, among others. ${ }^{5}$ However, the availability of this familial care is shifting rapidly as a result of social and demographic changes, both across Canada and globally. Increased longevity, declines in marriage and rises in divorce, childlessness and geographic mobility are leaving many seniors to enter later life alone, without aid in navigating complex systems of health and social care ${ }^{6-8}$ Recent estimates propose that up to 43.6 million older adults across the globe are spouseless and childless, and 4.4 million are spouseless, childless and without siblings. ${ }^{9}$ Even among older adults with living kin, many may lack someone to 
whom they are emotionally bonded, is geographically close at hand and is amenable to providing assistance. ${ }^{10}$

This dilemma was typified in Carney et $a l{ }^{11}{ }^{11}$ call to action to address the needs of this particularly vulnerable subset of the aging population, defining these 'elder orphans' as 'aged, community-dwelling older individuals who are socially and/or physically isolated without an available known family member or designated surrogate or caregiver'. In recent years, elder orphancy has increasingly drawn the attention of the medical community in light of its likely antecedence to older patients becoming 'unbefriended', reaching a state of cognitive decline such that they lack the ability to make health-related decisions, are without anyone to make these decisions in their stead and are without an advance directive. ${ }^{12}$ Emphasis within health research has been given to issues surrounding medical decision making and end-of-life care for unbefriended patients ${ }^{13-17}$; conversely, minimal effort has been made to identify and address the challenges faced by elder orphans while they are still residing within their communities and retain decision-making capacity. Of particular concern is the dearth of attention given to this population in the context of their ability to access needed health and social care services. ${ }^{18}$

Kinless and isolated older adults have been identified as being among society's most disadvantaged, ${ }^{10}$ faring poorly in both objective and subjective measures of socioeconomic, physiological and psychological health as compared with their with-kin counterparts. ${ }^{9}{ }^{19}$ Specifically, social isolation and loneliness leave older adults without family at a heightened risk of mental health issues such as depression, anxiety, and suicidality, ${ }^{20}$ dementia, ${ }^{21}$ and global cognitive decline ${ }^{22}$; furthermore, they are susceptible to an array of such chronic and acute physical conditions as coronary heart disease and stroke, ${ }^{23}$ decline in mobility ${ }^{24}$ and compromised motor and immune functioning, ${ }^{25}{ }^{26}$ among others. In an era in which the importance of ageing in place to well-being is well documented, ${ }^{27}$ kinlessness is inauspiciously predictive of institutionalisation ${ }^{28}$ and poorer quality of institutional care. ${ }^{29}$ As age-related chronic illnesses and impairments necessitate in-home assistance, transitional care and longterm planning, attempting to manage and coordinate the various aspects of their own care may prove especially discouraging for those older adults who are unable to depend on the aid of family or friends. These issues may be exacerbated through policy shifts and reforms promoting increasing reliance on families to support formalised care institutions. ${ }^{30}$

We will perform a systematic scoping review with the objective of assessing specific barriers and facilitators of health and social care access and system navigation experienced by elder orphans. Given the emergent nature of the topic of elder orphancy and the lack of a comprehensive review of work published to date in this subject area, a scoping review methodology has been chosen. While this approach is limited in that it does not involve a critical appraisal of individual studies selected for inclusion-which may vary in design, methodology and by extension, quality of results reported-a scoping review on this topic will be valuable in that it may identify gaps in knowledge, serve as an initial exploratory stage in the development of questions for further investigation and provide direction for future research priorities. ${ }^{31}$

\section{METHODS AND ANALYSIS \\ Protocol design}

This protocol was drafted in reference to the Preferred Reporting Items for Systematic Review and Meta-Analysis Protocols (PRISMA-P) ${ }^{32}$ and the PRISMA Extension for Scoping Reviews (PRISMA-ScR). ${ }^{33}$ The draft protocol has been reviewed by the research team members and revised as required. The final protocol has been registered with the Open Science Framework (https://osf.io/7vjty/).

\section{Study design}

This study will be completed using the scoping review methodology, a recently developed approach to knowledge synthesis used to map the foundational concepts in a research area as well as the principal sources and nature of the available evidence ${ }^{34}$ While broader in scope and inclusive of wider eligibility criteria than systematic reviews, scoping reviews still necessitate a rigorous and transparent methodological approach to ensure the credibility of their reported results. ${ }^{35}$

Arksey and O'Malley's ${ }^{36}$ six-staged methodological framework, which has further been developed by Levac et $a P^{37}$ and the Joanna Briggs Institute, ${ }^{38}$ will be used to guide the review process. The following steps will be employed accordingly: (1) identifying the research question(s); (2) identifying relevant literature; (3) study selection based on clear inclusion and exclusion criteria; (4) charting the data using standardised extraction tables; and (5) collating, summarising and reporting the results. The framework's optional consultation exercise (stage 6 ) is not planned. The selection process for the scoping review is presented in figure 1 .

\section{Stage 1: identifying the research question}

In line with the exploratory nature and focus on summarising breadth of evidence of the scoping review methodology, a broad research question was developed, namely, 'What are the barriers encountered by older adults aging without familial/informal support, or elder orphans, in accessing needed services and navigating current health and social care systems?'. This review will further seek to answer the subquestion: 'What are potential facilitating factors in identifying and addressing this population's barriers in accessing defined services?'.

For the purposes of this review, 'access to health and social care services' may comprise a range of conceptualisations, including but not limited to definitions encompassing the availability, affordability, acceptability and degree of accommodation of needed services ${ }^{39} 40$ and the ease or difficulty with which persons may use care services 


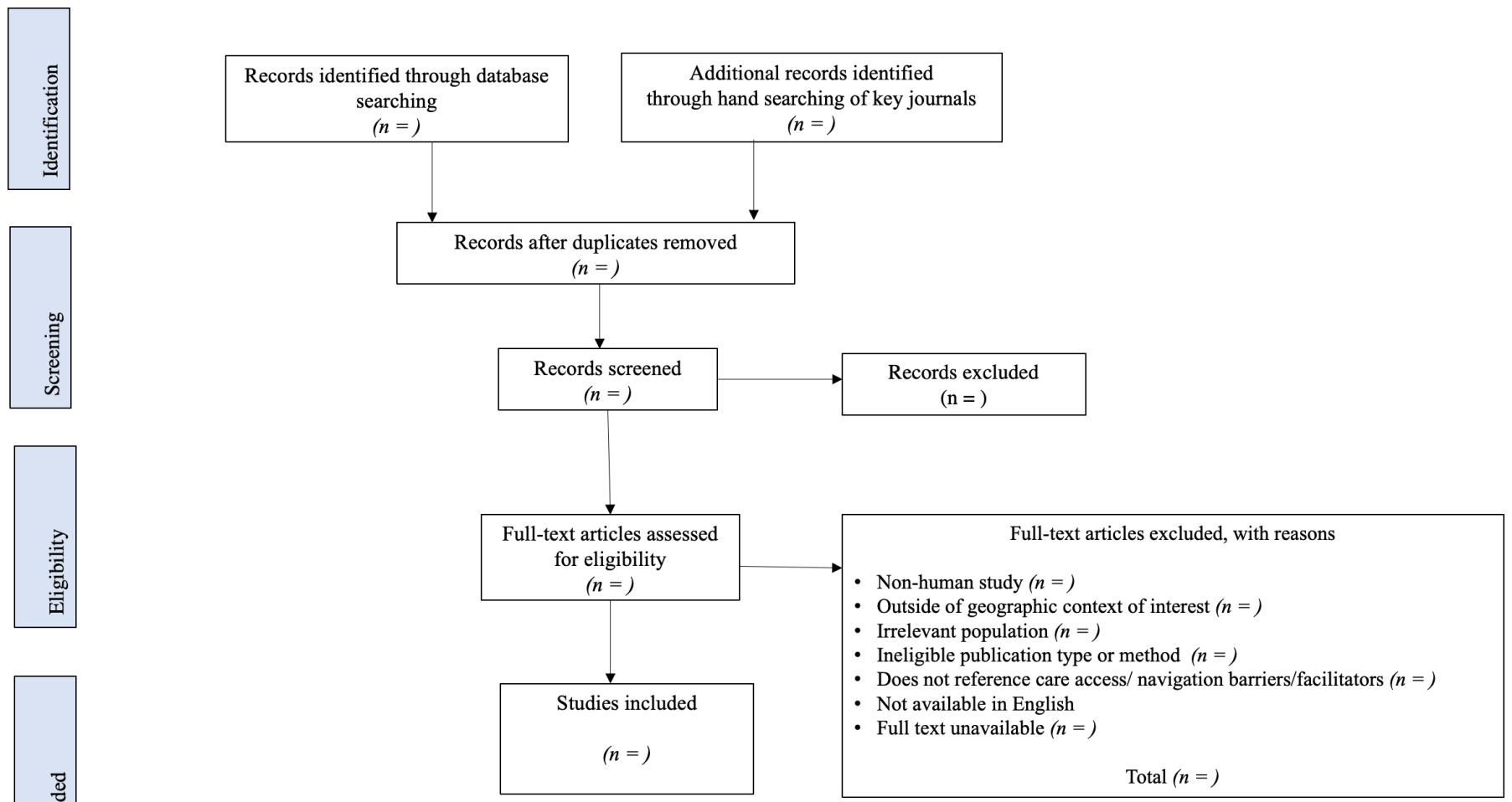

Figure 1 PRISMA flow diagram for the scoping review process. PRISMA, Preferred Reporting Items for Systematic Review and Meta-Analysis.

and resources that are compatible with their needs. ${ }^{41}$ The opportunity for members of vulnerable populations to gain access-and the extent of their access-to needed health and social care services exists not only if the services are available and adequately supplied; equitable access is further dependent on the relevancy and efficacy of those services and may be constrained by a multitude of organisational, demographic, social, economic, geographic and/or cultural barriers. ${ }^{42}$ Specifically, barriers within this context will be considered as factors that prevent an individual from gaining access to services or resources needed for the sustainment or betterment of their health, ${ }^{39}$ and facilitators as those that may improve an individual's ability to gain access to the aforementioned services. Both barriers and facilitators may be expressed as, but are not limited to, beliefs, experiences, expectations, attitudes or descriptions of behaviours, processes or outcomes.

\section{Stage 2: identifying relevant studies}

Identification of studies relevant to this review will be achieved through a systematic search of published literature through the following electronic bibliographic databases: CINAHL Complete, ASSIA, MEDLINE/ Pubmed, Scopus, Web of Science and PsycINFO. We will develop literature search strategies using text words and medical headings related to the population and concept of interest. The search strategy will be piloted to check the appropriateness of search terms and databases; the proposed search strategy is presented in table 1 . The search strategy will be adapted to each data source and terms will be searched as both keywords in the title and/ or abstract and subject headings as appropriate.

The database search will be limited to studies from January 2005 to the date of commencement; this constraint will be implemented to ensure that the literature reviewed is relevant to the current health and social care context and in consideration of the term 'elder orphan' being first used in the early 2000s in lay press publications ${ }^{43} 44$ and a nursing journal article. ${ }^{45}$ The geographic context for the search will be limited to developed countries including Canada, USA, Australia and New Zealand, and those of Western Europe and the UK as income, standard of living and health-related infrastructures within these nations may be comparable and therefore influence patterns of care access and inform response strategies. While older adults living in institutions and care facilities may additionally experience significant barriers in health and social care access and system navigation, this review will only examine those barriers and facilitators of access experienced by community-dwelling older adults. The decision to implement this limitation was based on several factors beyond alignment with Carney et $a l \mathrm{~s}^{11}$ definition of elder orphans. This limitation will increase the feasibility and quality of this review; limiting inclusion to studies with community-dwelling samples will enable a more in-depth and meaningful synthesis of a smaller number of studies relevant to the topic of interest. In addition, this review will be limited to the 


\begin{tabular}{|c|c|c|c|}
\hline & \multicolumn{2}{|l|}{ Key concepts } & \multirow{2}{*}{$\begin{array}{l}\text { Search terms } \\
\text { "elder* orphans", "adult orphans", "orphan* older person", "solo age*”, "unattached } \\
\text { older adult*”, "never married older adult*”, "unbefriended older adult*”, "kinless older } \\
\text { adult*”, "unbefriended elder", "isolated older adult*”, "isolated elder*”, "ageing alone" }\end{array}$} \\
\hline & $\begin{array}{l}\text { Isolated older } \\
\text { adults without } \\
\text { familial/informal } \\
\text { support }\end{array}$ & OR & \\
\hline AND & $\begin{array}{l}\text { Barriers/ } \\
\text { facilitators in } \\
\text { navigation/ } \\
\text { service access }\end{array}$ & OR & 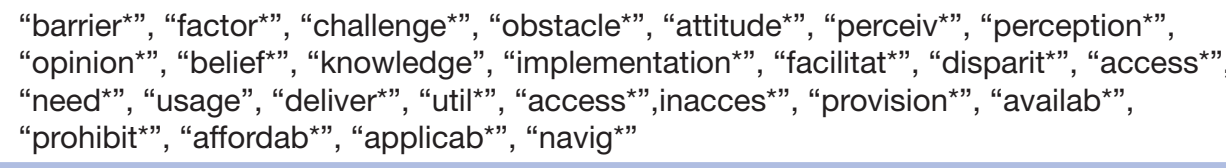 \\
\hline AND & $\begin{array}{l}\text { Health/social } \\
\text { care and } \\
\text { services }\end{array}$ & OR & $\begin{array}{l}\text { "health", "healthcare", "health care", "primary care", secondary care", tertiary care", } \\
\text { "emergency care", "community healthcare", "service", "program*”, "resource*”, } \\
\text { "treatment*”, "intervention*”, "strateg*”, "refer"”, "consult"”, "social care", "social } \\
\text { work*”, "home care", "voluntary care service*”, "community care", "social service*” }\end{array}$ \\
\hline
\end{tabular}

${ }^{\star}$ This symbol represents unlimited searches for variations on a word that are formed with different suffixes.

study of community-dwelling elder orphans in recognition of the barriers to care access and system navigation that may differ for this subpopulation in comparison with their institutionalised counterparts. Furthermore, we have chosen to implement this constraint in order to facilitate identification of barriers or issues experienced by this population while living within the community which may in part lead to their early institutionalisation. Only studies published in English will be included due to the linguistic abilities of the reviewers.

Search results will be downloaded into a citation management system where the articles will be screened for inclusion and duplicates removed. The electronic search will be documented by stating the date, keyword search, database used, number of publications retrieved and number of publications selected. The reference lists of the articles included in the final analysis will also be scanned to identify additional relevant references and ensure that no key articles were missed. Hand-searching of key journals will also be conducted to identify articles that have been missed in database and reference list searches. The authors note that the nature of the literature search in a scoping review is often an iterative process and that as the search is conducted additional keywords, sources and potentially useful search terms may be discovered and incorporated into the strategy. Any such changes will be documented to ensure transparency and auditability.

\section{Stage 3: study selection}

The selection of literature will occur in two phases. The first phase will involve screening of titles and abstracts of the identified studies from stage 2. Screening will be conducted independently by two reviewers using a structured screening form based on the identified eligibility criteria. To minimise the risk of bias, screening forms will be pilot tested by reviewers on a random selection of titles and abstracts to ensure consistency and reliability. Literature identified as potentially relevant will be passed to the second phase of screening. In phase 2 , the full text of potentially relevant studies will be retrieved and assessed thoroughly by the same reviewers using the inclusion criteria. Any disagreements between reviewers at each stage of the study selection process will be resolved through discussion, or if necessary, consultation with a third reviewer. Reasons for exclusion of full-text studies not meeting the inclusion criteria will be recorded and reported. The search results will be reported in full in the final scoping review. Although characteristics of individual studies, such as sample size or measures used, may influence the reliability of results, studies will not be excluded from the review based on their methodological quality if they meet the established inclusion criteria. This practice is in alignment with scoping review recommendations. ${ }^{36}{ }^{38}$ As noted in stage 5 , limitations of included studies will be documented and reported in the final review article. The process of study selection is reported using a PRISMA ${ }^{32}$ flow chart, which will be updated once the review is completed (figure 1).

\section{Eligibility criteria}

Studies of varied methodologies and designs (ie, qualitative, quantitative, mixed method and cross-sectional studies) will be considered for inclusion in this review. To be considered for inclusion, studies must meet the following criteria: (1) reference to barriers and/or facilitators to access or navigation of health and/or social care services and systems by the population of interest (elder orphans), (2) date of publication from January 2005 to the date of review commencement, (3) publication in English and (4) is a full report of study published in a peer-reviewed journal. Articles outside of those defined in the inclusion list, such as book reviews, opinion articles, commentaries or editorial reviews, will be excluded from the review; conference abstracts, theses or dissertations and review articles such as systematic reviews, metaanalyses, literature reviews, evidence syntheses, reviews of reviews, narrative reviews and critical reviews will also be excluded; however, their reference lists may be searched to ensure literature saturation. Eligibility of studies will also be determined based on their inclusion of participants 
aged 65 years or older who are identified as being without access to informal/familial support, are socially and/or physically isolated and are living within the community. Participants who live alone and have support visits from paid carers are included within this population. Studies involving only participants whose primary residence is an environment providing impromptu access to paid caregivers or staff 24 hours per day, such as in extra-care accommodation or assisted living facilities, non-human studies and studies focusing on any other age groups (eg, children) or caregivers or health and social care professionals or any other group will be excluded. In circumstances in which publications are identified that examine both elder orphans and other populations concurrently, only data and/or findings specific to the elder orphan portion of the study sample will be extracted and included in the review and synthesis. Such publications will only be included in the review on the condition that data and/or findings are presented in a way that information specific to only the elder orphan portion of the sample may be clearly identified and extracted; if publications do not present data and/or findings in such a way that information specific to only the elder orphan portion of the sample may be clearly identified, they will be extracted from the review. Any such publications will be flagged by reviewers during the screening stages of the review process, and any inter-reviewer disagreements regarding publication eligibility for inclusion in the review will be resolved through discussion and, if necessary, consultation with a third reviewer.

\section{Stage 4: charting the data}

Following studies being identified for inclusion in the review, a PRISMA ${ }^{32}$ flow diagram will be used to report the final numbers. A data extraction form from the Joanna Briggs Institute ${ }^{38}$ will be adapted and used to chart the following information from each article included in the review: author(s), year and journal of publication, country of study, study objectives/aims, type, methodology, and design, general characteristics of study participants, indicators of barriers to care access and navigation identified, and any other key findings that relate to the research question. To ensure common understanding of the extraction tool, we will organise a trial data charting during which both reviewers chart the same studies $(10 \%$ of all identified studies) and compare the resulting findings. Following the trial, each reviewer will work independently with the remaining studies. Any disagreements between the reviewers will be modified as necessary during the data extraction process, and any modifications will be documented and included in the final scoping review report. If an article is missing information regarding the intervention, outcomes or results, this will be noted in the data extraction. No data will be imputed. If duplicate publications are identified during data collection, they will be removed. If there are multiple reports from a single study, data from the study will be collated either by extracting data from each study separately and then combining the information from multiple data extraction forms, or extracting data from all articles into one single data extraction form, depending on the form of each article.

\section{Stage 5: data summary and synthesis of results}

As the scoping review methodology is primarily used to map the concepts underpinning a research area and the main sources and types of evidence available, the findings will provide an overview of the research rather than an assessment of the quality of individual studies. A risk of bias (quality) assessment will therefore not be conducted as part of this review. This decision is consistent with the framework proposed by Arksey and O'Malley, ${ }^{36}$ as well as the Joanna Briggs Institute methodological guidance for scoping reviews. ${ }^{38}$ However, we will include a summary of literature limitations identified in articles included in the review.

To present an overview of information retrieved and report the extent and nature of the literature, we will present results using two strategies: (1) a numerical overview of the amount, type and distribution of the included literature; and (2) a narrative synthesis and mapping of the results. The number of studies identified and selected along with the number of articles excluded at phase 1 and phase 2 of screening, and the reasons for exclusion that occurred at phase 2 of screening, will be presented in a PRISMA ${ }^{32}$ flow diagram. Results will be summarised in a tabulated format and will be accompanied by an in-depth narrative discussion mapping the findings from the extracted data and describing their relevance to the review's objective and research question. Finally, knowledge gaps and topics for future research will be identified.

\section{Patient and public involvement}

As identifying the barriers experienced by kinless and isolated elderly patients in accessing the health and social care services they need and navigating current systems of care will enable the amelioration of care practices and delivery, patients will eventually benefit from the body of knowledge this study contributes to. However, no patients or members of the public were involved in the protocol design or exploratory analyses, nor do we plan to include them.

\section{DISCUSSION}

Those individuals or groups considered most vulnerable are also those most adversely impacted by inequitable care access. Factors associated with the experience of barriers in access, both clinical, social and otherwise in nature, may accumulate and interact to influence one's ability to use needed services; in other words, those populations whose vulnerability arises via multiple pathways, characteristics and processes may encounter the greatest challenges in securing the resources they need. ${ }^{46}$ In addition, barriers may be experienced at various stages of the care-seeking process ${ }^{48}$ and may not be intuitively 
identifiable $^{49}$ or easily overcome. Insufficiently supported individuals may be left to 'slip through the cracks' into cycles of inadequate receipt of care and subsequent health-related decline. Kinlessness, isolation and their associated determinants place elder orphans at a severe disadvantage in regard to their physiological, social and psychological well-being. These circumstances may reinforce disparities in care access and patterns of service utilisation that result in unmet needs, deteriorating health outcomes and heightened healthcare costs. For example, barriers in care access for various populations have been associated with escalated rates of emergency service use for non-emergency reasons, ${ }^{50}$ mismanagement of chronic conditions, ${ }^{51}$ medication nonadherence,${ }^{52}$ misdiagnosis and/or delayed treatment, ${ }^{53}$ and preventable hospitalisations. ${ }^{55}$

Through a systematic search, mapping and synthesis of available literature, we will identify and describe the barriers encountered by elder orphans in both access of needed health and social care services and navigation of current systems of care. We will additionally establish potential facilitating factors in identifying and addressing this population's barriers in accessing defined services. To the best of the authors' knowledge, this review will be the first of its kind conducted on the topic of interest. While limited in that it will not assess the quality and risk of bias of included literature, a scoping review on this topic will contribute to an emerging, neglected area of research and bring attention to a growing, vulnerable and overlooked subset of the elderly population. Gaps in knowledge will be identified and recommendations for future areas of research will be made, laying the foundation for more in-depth analyses within specific care contexts. Identification of the key barriers experienced by this population in accessing the care and services they require may inform the development of supportive initiatives, such as patient advocacy and navigation programmes, peer networks and subsidised service provision; furthermore, it may highlight areas of health and social care policy in need of reform and/or the need for new policies better suited to an unprecedented context in which family members may no longer be relied on as primary caregivers for the elderly.

This review will be conducted in recognition of elder orphancy being an early stage in a problematic trajectory towards potentially avoidable early institutionalisation, health-related decline and the state of the unbefriended or surrogate-less patient. Intervention may be possible through establishing the barriers and issues experienced in health and social care access while this population still resides within their communities and retains decisionmaking capacity. Expected growth in the prevalence of elder orphancy and its implications for public health and demands for institutional care substantiates the need for efforts on behalf of researchers, policy makers and health and social care authorities to identify and address key issues experienced by these older adults in accessing needed services, and to develop responsive and comprehensive strategies to provide adequate resources and supports to promote their well-being, independence and engagement within their communities as long as possible.

Acknowledgements The authors gratefully acknowledge the helpful comments of their peer reviewers.

Contributors LK conceptualised the study, designed and prepared the draft of the review protocol, and is the guarantor. KT, RC, IR and TC contributed to refining the search strategy and eligibility criteria and editing the initial draft. TC acted as senior researcher and provided theoretical and methodological guidance. All authors further revised the manuscript and approved the final text.

Funding The authors have not declared a specific grant for this research from any funding agency in the public, commercial or not-for-profit sectors.

\section{Competing interests None declared.}

Patient and public involvement Patients and/or the public were not involved in the design, or conduct, or reporting, or dissemination plans of this research.

Patient consent for publication Not required.

Provenance and peer review Not commissioned; externally peer reviewed.

Open access This is an open access article distributed in accordance with the Creative Commons Attribution Non Commercial (CC BY-NC 4.0) license, which permits others to distribute, remix, adapt, build upon this work non-commercially, and license their derivative works on different terms, provided the original work is properly cited, appropriate credit is given, any changes made indicated, and the use is non-commercial. See: http://creativecommons.org/licenses/by-nc/4.0/.

\section{ORCID iDs}

Lucy Kervin http://orcid.org/0000-0002-8711-2173

Kelly Teo http://orcid.org/0000-0003-2405-6466

Ryan Churchill http://orcid.org/0000-0001-6510-4810

Indira Riadi http://orcid.org/0000-0001-6474-5384

Theodore D Cosco http://orcid.org/0000-0002-9977-8888

\section{REFERENCES}

1 Chatters LM, Taylor HO, Nicklett EJ, et al. Correlates of objective social isolation from family and friends among older adults. Healthcare 2018;6:24.

2 Long MV, Martin P. Personality, relationship closeness, and loneliness of oldest old adults and their children. J Gerontol B Psychol Sci Soc Sci 2000;55:P311-9.

3 Thomas PA, Liu H, Umberson D. Family relationships and well-being Innov Aging 2017;1:igx025.

4 Schulz R. Research priorities in geriatric palliative care: informal caregiving. J Palliat Med 2013;16:1008-12.

5 Schulz R, Eden J. Families caring for an aging America. Washington, DC: The National Academies Press, 2016.

6 Phillipson C. Ageing. Cambridge, UK: Polity Press, 2013.

7 Redfoot D, Feinberg L, Houser AN. The aging of the baby boom and the growing care gap: a look at future declines in the availability of family caregivers. Washington, DC: AARP Public Policy Inst, 2013.

8 Roth DL, Fredman L, Haley WE. Informal caregiving and its impact on health: a reappraisal from population-based studies. Gerontologist 2015;55:309-19.

9 Verdery AM, Margolis R, Zhou Z, et al. Kinlessness around the world. J Gerontol B Psychol Sci Soc Sci 2019;74:1394-405.

10 Verdery AM, Margolis R. Projections of white and black older adults without living kin in the United States, 2015 to 2060. Proc Natl Acad Sci U S A 2017;114:11109-14.

11 Carney MT, Fujiwara J, Emmert BE, et al. Elder orphans hiding in plain sight: a growing vulnerable population. Curr Gerontol Geriatr Res 2016;2016:1-11.

12 Chamberlain S, Baik S, Estabrooks C. Going it alone: a scoping review of unbefriended older adults. Can J Aging 2018;37:1-11.

13 High DM. Old and alone: surrogate health care decision-making for the elderly without families. J Aging Stud 1990;4:277-88.

14 Pope TM. Making medical decisions for patients without surrogates. N Engl J Med 2013;369:1976-8.

15 Weiss BD, Berman EA, Howe CL, et al. Medical decision-making for older adults without family. J Am Geriatr Soc 2012;60:2144-50. 
16 White DB, Curtis JR, Wolf LE, et al. Life support for patients without a surrogate decision maker: who decides? Ann Intern Med 2007; 147:34-40.

17 White DB, Curtis JR, Lo B, et al. Decisions to limit life-sustaining treatment for critically ill patients who lack both decisionmaking capacity and surrogate decision-makers. Crit Care Med 2006;34:2053-9.

18 Montayre J, Montayre J, Thaggard S. The elder orphan in healthcare settings: an integrative review. J Popul Ageing 2019;12:515-32.

19 Waite L, Das A. Families, social life, and well-being at older ages. Demography 2010;47 Suppl:S87-109.

20 Beutel ME, Klein EM, Brähler E, et al. Loneliness in the general population: prevalence, determinants and relations to mental health. BMC Psychiatry 2017;17:97.

21 Penninkilampi R, Casey A-N, Singh MF, et al. The association between social engagement, loneliness, and risk of dementia: a systematic review and meta-analysis. $J$ Alzheimers Dis 2018;66:1619-33.

22 Braak H, Del Tredici K. Alzheimer's disease: pathogenesis and prevention. Alzheimers Dement 2012;8:227-33.

23 Valtorta NK, Kanaan M, Gilbody S, et al. Loneliness and social isolation as risk factors for coronary heart disease and stroke: systematic review and meta-analysis of longitudinal observational studies. Heart 2016;102:1009-16.

24 Perissinotto CM, Stijacic Cenzer I, Covinsky KE. Loneliness in older persons: a predictor of functional decline and death. Arch Intern Med 2012;172:1078-83.

25 Buchman AS, Boyle PA, Wilson RS, et al. Loneliness and the rate of motor decline in old age: the rush memory and aging project, a community-based cohort study. BMC Geriatr 2010;10:77.

26 Uchino BN, Vaughn AA, Carlisle M. Social support and immunity. In: Segerstrom S, ed. The Oxford Handbook of Psychoneuroimmunology. New York, NY: Oxford Press, 2012: 214-33.

27 Marek KD, Popejoy L, Petroski G, et al. Clinical outcomes of aging in place. Nurs Res 2005;54:202-11.

28 Thomeer MB, Mudrazija S, Angel JL. Relationship status and longterm care facility use in later life. J Gerontol B Psychol Sci Soc Sci 2016;71:711-23.

29 Gaugler JE, Anderson KA, Zarit SH, et al. Family involvement in nursing homes: effects on stress and well-being. Aging Ment Health 2004;8:65-75.

30 Torjman S. Policy innovations for an aging Society. Toronto, ON: Maytree Foundation, 2018.

31 Colquhoun HL, Levac D, O'Brien KK, et al. Scoping reviews: time for clarity in definition, methods, and reporting. J Clin Epidemiol 2014;67:1291-4.

32 Moher D, Shamseer L, Clarke M, et al. Preferred reporting items for systematic review and meta-analysis protocols (PRISMA-P) 2015 statement. Syst Rev 2015;4:1.

33 Tricco AC, Lillie E, Zarin W, et al. PRISMA extension for scoping reviews (PRISMA-ScR): checklist and explanation. Ann Intern Med 2018;169:467-73.
34 Tricco AC, Lillie E, Zarin W, et al. A scoping review on the conduct and reporting of scoping reviews. BMC Med Res Methodol 2016;16:15

35 Munn Z, Peters MDJ, Stern C, et al. Systematic review or scoping review? Guidance for authors when choosing between a systematic or scoping review approach. BMC Med Res Methodol 2018;18:143.

36 Arksey H, O'Malley L. Scoping studies: towards a methodologica framework. Int J Soc Res Methodol 2005;8:19-32.

37 Levac D, Colquhoun H, O'Brien KK. Scoping studies: advancing the methodology. Implement Sci 2010;5:69.

38 Peters MDJ, Godfrey C, Mclnerney P. Chapter 11: Scoping Reviews (2020 version). In: Aromataris E, Munn Z, eds. JBI manual for evidence synthesis, 2020.

39 Gulliford M, Figueroa-Munoz J, Morgan M, et al. What does 'access to health care' mean? J Health Serv Res Policy 2002;7:186-8.

40 Penchansky R, Thomas JW. The concept of access: definition and relationship to consumer satisfaction. Med Care 1981;19:127-40.

41 Richard L, Furler J, Densley K, et al. Equity of access to primary healthcare for vulnerable populations: the impact international online survey of innovations. Int J Equity Health 2016;15:64.

42 Bhatt J, Bathija P. Ensuring access to quality health care in vulnerable communities. Acad Med 2018;93:1271-5.

43 Kunerth J. Seniors Outliving family, becoming elder orphans, Chicago Tribune 2003.

44 Sherer R. Who will care for elder orphans. Geriatric Times 2004

45 Varner J. The elder orphans: who are they? Ala Nurse 2005;32:19-21.

46 Corscadden L, Levesque JF, Lewis V, et al. Factors associated with multiple barriers to access to primary care: an international analysis. Int J Equity Health 2018;17:28.

47 Shippee ND, Shah ND, May CR, et al. Cumulative complexity: a functional, patient-centered model of patient complexity can improve research and practice. J Clin Epidemiol 2012;65:1041-51.

48 Carrillo JE, Carrillo VA, Perez HR, et al. Defining and targeting health care access barriers. J Health Care Poor Underserved 2011;22:562-75.

49 Williams MM. Invisible, unequal, and forgotten: health disparities in the elderly. Notre Dame Journal Law Ethics Public Policy;21:441-78.

50 Rust G, Ye J, Baltrus P, et al. Practical barriers to timely primary care access: impact on adult use of emergency department services. Arch Intern Med 2008;168:1705-10.

51 Hossain WA, Ehtesham MW, Salzman GA, et al. Healthcare access and disparities in chronic medical conditions in urban populations. South Med J 2013;106:246-54.

52 Roumie CL, Greevy R, Wallston KA, et al. Patient centered primary care is associated with patient hypertension medication adherence. $J$ Behav Med 2011;34:244-53

53 de Moissac D, Bowen S. Impact of language barriers on quality of care and patient safety for official language minority francophones in Canada. J Patient Exp 2019;6:24-32.

54 Cassim S, Chepulis L, Keenan R, et al. Patient and carer perceived barriers to early presentation and diagnosis of lung cancer: a systematic review. BMC Cancer 2019;19:25

55 Rizza P, Bianco A, Pavia M, et al. Preventable hospitalization and access to primary health care in an area of southern Italy. BMC Health Serv Res 2007;7:134. 